20. London, F. Superfluids (Wiley, New York, 1950).

21. Tokuyasu, T. A., Hess, D. W. \& Sauls, J. A. Vortex states in an unconventional superconductor and the mixed states of UPt ${ }_{3}$. Phys. Rev. B 41, 8891-8903 (1990).

22. Luk'yanchuk, I. A. \& Zhitomirsky, M. E. Magnetic properties of unconventional superconductors. Supercond. Rev. 1, 207-256 (1995).

23. Yaron, P. L. et al. Neutron diffraction studies of flowing and pinned magnetic flux lattices in $2 \mathrm{H}-$ $\mathrm{NbSe}_{2}$. Phys. Rev. Lett. 73, 2748-2751 (1994).

24. Brandt, E. H. Precision Ginzburg-Landau solution of ideal vortex lattices for any induction and symmetry. Phys. Rev. Lett. 78, 2208-2211 (1997).

25. Broholm, C. et al. Anisotropic temperature dependence of the magnetic-field penetration in superconducting $\mathrm{UPt}_{3}$. Phys. Rev. Lett. 65, 2062-2065 (1990).

26. Yaouanc, A. et al. Strong axial anisotropy of the magnetic penetration length in superconducting $\mathrm{UPt}_{3 .}$ J. Phys. Condens. Matter 10, 9791-9798 (1998).

27. Ellman, B., Taillefer, L. \& Poirier, M. Transverse ultrasound revisited: a directional probe of the A phase in UPt 3 . Phys. Rev. B 54, 9043-9046 (1996).

28. Clem, J. R. Simple model for the vortex core in a type II superconductor. J. Low Temp. Phys. 18, 427 434 (1974).

29. Taillefer, L. \& Lonzarich, G. G. Heavy fermion quasi-particles in UPt ${ }_{3}$. Phys. Rev.Lett. 60, 1570-1573 (1988).

30. Hayden, S. M., Taillefer, L., Vettier, C. \& Flouquet, J. Antiferromagnetic order in UPt ${ }_{3}$ under pressure: Evidence for a direct coupling to superconductivity. Phys. Rev. B 46, 8675-8678 (1992).

31. Aeppli, G. et al. Magnetic order and fluctuations in superconducting UPt 3 . Phys. Rev. Lett. 60, 615618 (1988).

32. Park, K. A. \& Joynt, R. Phase diagram of $\mathrm{UPt}_{3}$ in the $\mathrm{E}_{1 \mathrm{~g}}$ model. Phys. Rev. Lett. 74, 4734-4737 (1995).

Correspondence and requests for materials should be addressed to A.H. (e-mail: huxley@drfmc.ceng.cea.fr).

\section{Shaped-pulse optimization of coherent emission of high-harmonic soft X-rays}

\author{
R. Bartels ${ }^{\star}$, S. Backus ${ }^{\star}$, E. Zeek ${ }^{\star}$, L. Misoguti ${ }^{\star}$, G. Vdovin $†$, I. P. Christov $\ddagger$, \\ M. M. Murnane ${ }^{\star}$ \& H. C. Kapteyn ${ }^{\star}$
}

* JLA and Department of Physics, University of Colorado and National Institute of Standards and Technology, Campus Box 440, Boulder, Colorado 80309-0440, USA

$\dagger$ Delft University of Technology, 2600 GA Delft, The Netherlands

$\$$ Department of Physics, Sofia University, Sofia, Bulgaria

When an intense laser pulse is focused into a gas, the light-atom interaction that occurs as atoms are ionized results in an extremely nonlinear optical process ${ }^{1-3}$ - the generation of high harmonics of the driving laser frequency. Harmonics that extend up to orders of about 300 have been reported ${ }^{4,5}$, some corresponding to photon energies in excess of $500 \mathrm{eV}$. Because this technique is simple to implement and generates coherent, laser-like, soft $X$-ray beams, it is currently being developed for applications in science and technology; these include probing the dynamics in chemical and materials systems ${ }^{6}$ and imaging ${ }^{7}$. Here we report that by carefully tailoring the shape $e^{8}$ of intense light pulses, we can control ${ }^{9,10}$ the interaction of light with an atom during ionization, improving the efficiency of X-ray generation by an order of magnitude. We demonstrate that it is possible to tune the spectral characteristics of the emitted radiation, and to steer the interaction between different orders of nonlinear processes.

This work builds on a number of other recent advances in laser and nonlinear-optical science and technology. The use of temporally shaped ultrashort pulses to create 'designer' atomic wavepackets $^{11}$, to control two-photon absorption ${ }^{12,13}$, and to control molecular processes ${ }^{11,14}$ has been a topic of increasing interest as laser technology has developed. Recent work has demonstrated techniques to increase the conversion efficiency of high-harmonic generation by using waveguide ${ }^{15}$ and new focusing geometries ${ }^{16,17}$, and very short duration ( $\leq 25 \mathrm{fs}$, or $\sim 10$ optical cycles) light pulses $^{18}$. It has also been shown that the temporal ordering of

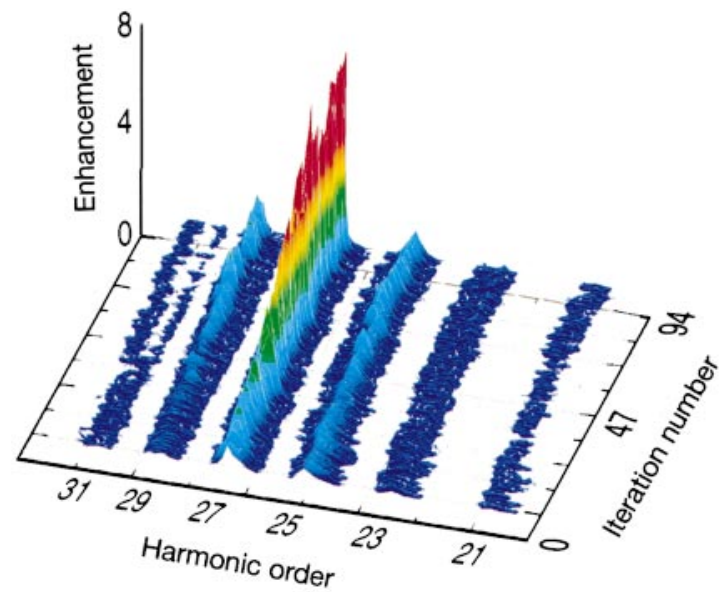

Figure 1 Optimization of a single (27th) harmonic in argon while suppressing adjacent harmonics. The optimization criterion (fitness function) for this run corresponded to $f=\Sigma s_{j}-\Sigma s_{l}-\Sigma s_{k}$, where $s$ corresponds to the signal level of a CCD pixel, and the ranges $i, j$ and $k$ represent the pixels corresponding to a $0.5-\mathrm{nm}$ spectral bandwidth centred around the 25th, 27th and 29th harmonic. The peak enhancement for the 27th harmonic is a factor of 8 , while the energy enhancement is a factor of 4.6. The contrast ratio between the 27 th harmonic and adjacent harmonic increases by a factor of 4 .

colours in the light pulse affects the spectral characteristics of the high-harmonic radiation ${ }^{19,20}$. However, that work was limited to simple adjustment of the light pulse 'chirp' and did not reveal any ability to selectively control the high harmonic generation (HHG) process or to improve overall conversion efficiency.

The present work uses a newly developed laser system that generates high-power ultrafast laser pulses with the capability of temporally reshaping them in a very precise and flexible manner ${ }^{21}$. We show that very subtle changes (resulting in changes in the time duration of the light pulse of only a few femtoseconds) can manipulate the electronic response of an atom, and thereby control the spectral characteristics and brightness of the soft X-ray coherent radiation. We have found this effect to be very general, occurring over a wide range of parameters and in different gases. In light of past work, the effectiveness of this technique is unexpected. It illustrates significant differences between low-order nonlinear processes such as second-harmonic generation and high-order processes such as HHG that are inherently non-perturbative. Furthermore, under some conditions it is possible to channel most of the high-harmonic emission into a single spectral peak creating a coherent, nearly monochromatic soft X-ray source with a greatly enhanced brightness.

In our experiment, we focus light pulses from an ultrafast laser system with a repetition rate of $1 \mathrm{kHz}$ and a pulse energy of up to $1 \mathrm{~mJ}$ into a $175-\mu \mathrm{m}$ diameter, gas-filled capillary waveguide. The pulse peak intensity $\left(\sim 2 \times 10^{14} \mathrm{~W} \mathrm{~cm}^{-2}\right)$ is sufficient to field-ionize the atoms (typically argon), in the process generating high-harmonic extreme ultraviolet (XUV) radiation. The capillary waveguide allows us to create an extended region of high laser intensity and long coherence length to efficiently generate the high-harmonic radiation $^{15}$, which is observed using an X-ray charge-coupled device (CCD) camera directly coupled to a grazing-incidence spectrometer.

The laser system incorporates a micromachined deformable mirror pulse-shaping apparatus ${ }^{22}$. This adaptive optic element is used in a position within the laser system where the frequency components of the light pulse are spatially separated and can change the relative path length of the various colours, thus reordering the arrival times of the colours of the short pulse. The light pulse bandwidth is about $80 \mathrm{~nm}$ full-width at half-maximum (FWHM) centred at $800 \mathrm{~nm}$, and is not altered by the pulse shaper. 


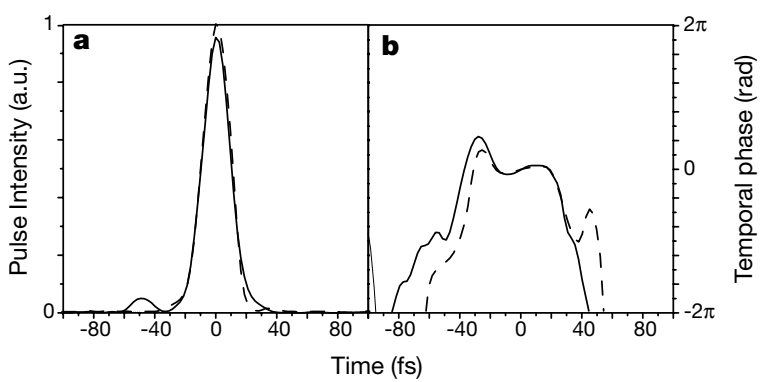

Figure 2 Laser pulse characteristics. $\mathbf{a}, \mathbf{b}$, Amplitude (a) and phase (b) of the transformlimited (dashed line) and optimized (solid line) laser pulses corresponding to Fig. 1. These data were taken using the frequency-resolved optical gating technique (FROG). The energy of the pulse is kept constant.

We have shown that this mirror, when combined with adaptive control using an evolutionary search procedure, can allow us to generate nearly perfectly transform-limited, 15 -fs $(<6$ optical cycles) light pulses from the laser ${ }^{21}$. To do so, we optimized the intensity of second-harmonic radiation (SHG) generated by the laser pulses passing through a thin frequency-doubling crystal $^{23}$. The actual laser pulse shapes were characterized in both amplitude and phase using the frequency-resolved optical gating (FROG) technique $^{24}$. In the case of SHG-optimized control, the highest conversion efficiency was observed to correspond to the highest peak power obtainable for a given pulse spectrum (that is, the timebandwidth or Fourier-transform limit of laser pulse, where all frequency components arrive at once). This is the intuitive result, since a nonlinear-optical process should be most efficient at the highest peak intensity.

For our experiments to optimize HHG, we started with an 18 -fs, Fourier-transform-limited laser pulse obtained using the SHG optimization technique. The initial curve of Fig. 1 (iteration number 0 ) shows the HHG spectrum from argon gas at about the 27 th harmonic, using a transform-limited 18 -fs pulse. This curve also represents the highest overall signal level obtainable before learning optimization of the HHG. We verified that adjustment of the linear chirp of the pulse did not improve the brightness of the HHG spectrum. In order to use the evolutionary strategy (ES) for HHG optimization, a spectral window of $0.5 \mathrm{~nm}$ surrounding the 27 th harmonic was selected.

The algorithm starts with a set of randomly selected pulse shapes (corresponding to 19 random voltages on the pulse shaper), and searches for a pulse shape to maximize the signal intensity here while at the same time minimizing the adjacent 25th and 29th order harmonics. The best (fittest) pulses are then randomly altered (mutated), by adding a normally distributed random variable to the value of each pixel, and retested. Each 'parent' solution generates five mutated 'children'. The algorithm converges to some optimal solution after approximately 50 iterations. Figure 1 shows the optimum solution after each iteration of the algorithm.

Surprisingly, after optimization the intensity of a single harmonic (27th) has been increased by a factor of eight, while adjacent harmonic orders are only slightly enhanced. The level of enhancement depends on the gas pressure, harmonic order and gas type, with the largest enhancements (of over an order of magnitude) observed at a gas pressure corresponding to macroscopic phasematching of the high-harmonic process, and for the harmonic (27th) where neutral gas absorption of the light is near the minimum. However, enhancement of more than three times is observed under virtually every set of conditions. As different optimization searches proceed, the relative heights of the various harmonics can change, and energy is channelled to the 27th harmonic as the search converges. The total integrated X-ray flux also increases by a factor of two to six, depending on the gas pressure and harmonic orders

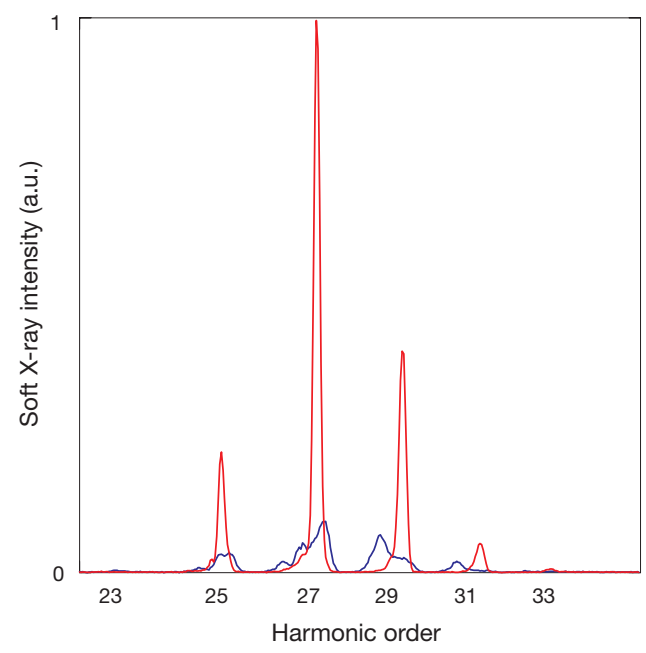

Figure 3 Optimization of a single harmonic in argon. The spectral window occurs at longer wavelengths than in Fig. 1 and without suppressing adjacent harmonics. That is, the fitness function is $f=\Sigma s_{j}$, where the range $j$ represents pixels to the 'red' side of the 27th harmonic. The harmonic peak is enhanced by over an order of magnitude. Harmonics before and after optimization are shown in blue and red respectively.

being optimized. For example, in the case of krypton gas, the combined nonlinearity and absorption characteristics of the gas result in about six harmonic peaks of comparable intensity before optimization. Optimization of any particular peak in the range of $17-23$ results in enhancement of this peak by a factor of three to four times, while harmonics immediately adjacent to the 'target' peak are enhanced by about 1.5-2 times, and other peaks have negligible or negative enhancement.

Figure 2 shows the laser pulse shapes corresponding to the transform-limited and final HHG spectra shown in Fig. 1. The transform-limited and optimized laser pulse shape are shown in Fig. $2 \mathrm{a}$, while Fig. $2 \mathrm{~b}$ shows the corresponding phase values. The optimized pulse has more structure and phase change on the leading edge, but is not much broader than the transform limit $(\sim 21.6 \mathrm{fs}$ compared with $18 \mathrm{fs}$ ). Thus, very slight modification of the driving pulse can result in drastic changes in the harmonic radiation generated.

We have found that different optimization runs, using the same fitness criteria, yield slightly differing pulse shapes even when similar enhancements are observed, suggesting that some degrees of freedom for the pulse shape are not significant, while others are; work is in progress to modify the fitness criteria to try to identify the critical features. The enhanced pre-pulse observed in the optimized pulse is not always present, and is probably a result of limitations of the phase-only pulse shaper; however, the presence of some phase structure on the leading edge of the pulse is a consistent feature. Furthermore, as the intensity of the pre-pulse is below that required for ionization and therefore HHG emission, it is unlikely to be important. By selecting different criteria (that is, different spectral windows, different harmonics, and/or different gases), the ES search algorithm can converge on different optimal solutions. Figure 3, for example, shows the results before (blue) and after (red) optimization of the 27th harmonic in argon, but in this case optimized over a shifted spectral window positioned to the left of the harmonic peak, with no suppression of adjacent peaks.

The optimization process increased both the peak intensity and spectral purity of the harmonic, increasing the peak by over an order of magnitude. Choice of the optimization window on the red or the blue side of the initial peak consistently results in a red or blue shift of the harmonic; however, the resulting spectral bandwidth varies. This optimization is consistent with previous work that showed that 
a positive (red to blue) chirp on the driving pulse can result in both a red shift and a spectral narrowing of the harmonic emission peaks ${ }^{19}$ (although in that case no dramatic increase in brightness or selectivity was observed). The measured width of the optimized 27 th harmonic is $0.24 \mathrm{eV}$, corresponding to our instrument resolution. Thus, the true peak and spectral enhancements may be even higher. Since the duration of the XUV pulse is probably somewhat shorter than the driving laser pulse, the resulting HHG spectrum corresponds to an XUV pulse much closer to the time-bandwidth limit than before optimization. This result illustrates quite clearly that pulse shaping can alleviate what had been thought to be a fundamental 'trade-off' for use of HHG as a light source - that the use of very short driving pulses, although dramatically increasing the efficiency of $\mathrm{HHG}$, results in a broader spectrum for the individual harmonic peaks.

To explain the unexpected result that very slight changes in laser pulse shape can dramatically enhance and select individual harmonics, we consider the semi-classical rescattering model of $\mathrm{HHG}^{25,26}$. From a classical point of view, electrons are ionized and accelerated away from the core during one half-cycle of the laser field, and can be subsequently driven back to the core when the laser field reverses. Some fraction of the ionized electrons can recombine with the parent ion and give off their energy in the form of high harmonics. This process occurs over a few optical cycles of the laser field, resulting in an approximately 5 -fs X-ray burst. Particular harmonics are generated by electrons returning to the core with a specific return energy that is related to the exact time within the optical cycles when the electron is initially ionized.

We believe that the adaptive optimization algorithm finds a pulse with the correct phase sequence to ensure that the continuum X-ray emission generated by a particular cycle of the laser pulse reinforces constructively or destructively with different parts of the continuum generated by adjacent cycles. This coherent control can lead to channelling and redirection of energy between different high-order nonlinear interactions.

These findings therefore demonstrate a new type of intra-atomic 'phase matching' between the laser field and the wavefunction of the ionized electron. Preliminary calculations based on phase-only pulse shaping confirm this interpretation. From a quantum point of view, our optimized laser pulse can adjust the quantum phase of the electron wavefunction which returns to the core, to optimize it for a particular harmonic feature. This work may lead to other new methods for control of highly nonlinear systems, as well as improving the utility of the HHG source for application experiments in a broad range of science $\mathrm{e}^{27-30}$.

Received 2 March; accepted 15 May 2000

1. McPherson, A. et al. Studies of multiphoton production of vacuum-ultraviolet radiation in the rare gasses. J. Opt. Soc. Am. B 4, 595-601 (1987).

2. Macklin, J. J., Kmetec, J. D. \& Gordon, C. L. III High-order harmonic generation using intense femtosecond pulses. Phys. Rev. Lett. 70, 766-769 (1993).

3. L'Huillier, A. \& Balcou, P. High-order harmonic generation in rare gases with a 1-ps $1053 \mathrm{~nm}$ laser. Phys. Rev. Lett. 70, 774-777 (1993).

4. Chang, Z., Rundquist, A., Wang, H., Murnane, M. M. \& Kapteyn, H. C. Generation of coherent X-rays at $2.7 \mathrm{~nm}$ using high harmonics. Phys. Rev. Lett. 79, 2967-2970 (1997).

5. Spielmann, C. et al. Generation of coherent X-rays in the water window using 5 -femtosecond laser pulses. Science 278, 661-664 (1997)

6. Rettenberger, A., Leiderer, P., Probst, M. \& Haight, R. Ultrafast electron transport in layered semiconductors studied with femtosecond-laser photoemission. Phys. Rev. B 56, 12092-12095 (1997).

7. Descamps, D. et al. Extreme ultraviolet interferometry measurements with high-order harmonics. Opt. Lett. 25, 135-137 (2000)

8. Wefers, M. \& Nelson, K. Analysis of programmable ultrashort waveform generation using liquidcrystal spatial light-modulators. J. Opt. Soc. Am. B 12, 1343-1362 (1995).

9. Judson, R. \& Rabitz, H. Teaching lasers to control molecules. Phys. Rev. Lett. 68, 1500-1503 (1992)

10. Warren, W., Rabitz, H. \& Dahleh, M. Coherent control of quantum dynamics: the dream is alive. Science 259, 1581-1589 (1993)

11. Weinacht, T. C., Ahn, J. \& Bucksbaum, P. H. Controlling the shape of a quantum wavefunction. Nature 397, 233-235 (1999).

12. Meshulach, D. \& Silberberg, Y. Coherent quantum control of two-photon transitions by a femtosecond laser pulse. Nature 396, 239-242 (1998).

13. Meshulach, D. \& Silberberg, Y. Coherent quantum control of multiphoton transitions by shaped ultrashort optical pulses. Phys. Rev. A 60, 1287-1292 (1999).
14. Assion, A. et al. Control of chemical reactions by feedback-optimized phase-shaped femtosecond laser pulses. Science 282, 919-922 (1998).

15. Rundquist, A. et al. Phase-matched generation of coherent soft X-rays. Science 280, 1412-1415 (1998)

16. Roos, L. et al. Controlling phase matching of high-order harmonic generation by manipulating the fundamental field. Phys. Rev. A 60, 5010-5018 (1999).

17. Altucci, C., Bruzzese, R., D'Antuoni, D., de Lisio, C. \& Solimeno, S. Harmonic generation in gases by use of Bessel-Gauss laser beams. J. Opt. Soc. Am. B 17, 34-42 (2000).

18. Zhou, J., Peatross, J., Murnane, M. M., Kapteyn, H. C. \& Christov, I. P. Enhanced high harmonic generation using 25 femtosecond laser pulses. Phys. Rev. Lett. 76, 752-755 (1996).

19. Chang, Z., Rundquist, A., Wang, H., Kapteyn, H. C. \& Murnane, M. M. Temporal phase control of soft-X-Ray harmonic emission. Phys. Rev. A 58, R30-R33 (1998).

20. Salieres, P., Antoine, P., de Bohan, A. \& Lewenstein, M. Temporal and spectral tailoring of high-order harmonics. Phys. Rev. Lett. 81, 5544-5547 (1998).

21. Zeek, E. et al. Adaptive pulse compression for transform-limited 15fs high-energy pulse generation. Opt. Lett. 25, 587-589 (2000).

22. Zeek, E. et al. Pulse compression using deformable mirrors. Opt. Lett. 24, $493-495$ (1999).

23. Yelin, D., Meshulach, D. \& Silberberg, Y. Adaptive femtosecond pulse compression. Opt. Lett. 22, 1793-1795 (1997).

24. DeLong, K. W., Trebino, R., Hunter, J. \& White, W. E. Frequency-resolved optical gating with the use of second-harmonic generation. J. Opt. Soc. Am. B 11, 2206-2215 (1994).

25. Kulander, K. C., Schafer, K. J. \& Krause, J. L. in Super-Intense Laser-Atom Physics (eds Piraux, B., L'Huillier, A. \& Rzazewski, K.) 95-110 (Plenum, New York, 1993).

26. Lewenstein, M., Balcou, P., Ivanov, M. Y. \& Corkum, P. B. Theory of high-harmonic generation of lowfrequency laser fields. Phys. Rev. A 49, 2117-2132 (1993).

27. Ditmire, T. et al. Nuclear fusion from explosions of femtosecond laser-heated deuterium clusters. Nature 398, 489-492 (1999).

28. LeBlanc, S. P. et al. Temporal characterization of a self-modulated laser wakefield. Phys. Rev. Lett. 77, 5381-5384 (1996)

29. de Boeij, W. P., Pshenichnikov, M. S. \& Wiersma, D. A. Ultrafast solvation dynamics explored by femtosecond photon echo spectroscopies. Ann. Rev. Phys. Chem. 49, 99-123 (1998).

30. Schoenlein, R. W. et al. Femtosecond X-ray pulses at 0.4 angstrom generated by 90 degrees Thomson scattering: A tool for probing the structural dynamics of materials. Science 274, 236-238 (1996).

\section{Acknowledgements}

We gratefully acknowledge support for this work from the Department of Energy and the National Science Foundation.

Correspondence and requests for materials should be addressed to H. C. K. (e-mail: kapteyn@jila.colorado.edu).

\section{Configurational entropy and diffusivity of supercooled water}

\section{Antonio Scala ${ }^{\star} \dagger$, Francis W. Starr ${ }^{\star} \dagger$, Emilia La Nave*, Francesco Sciortino $\neq \&$ H. Eugene Stanley ${ }^{\star}$}

* Center for Polymer Studies, Center for Computational Science, and Department of Physics, Boston University, Boston, Massachusetts 02215, USA $\ddagger$ Dipartimento di Fisica e Istituto Nazionale per la Fisica della Materia, Universitá di Roma La Sapienza, P.le Aldo Moro 2, I-00185, Roma, Italy

As a liquid approaches the glass transition, its properties are dominated by local potential minima ${ }^{1,2}$ in its energy landscape. The liquid experiences localized vibrations in the basins of attraction surrounding the minima, and rearranges via relatively infrequent inter-basin jumps ${ }^{3}$. As a result, the liquid dynamics at low temperature are related to the system's exploration of its own configuration space. The 'thermodynamic approach' to the glass transition considers the reduction in configuration space ${ }^{4-8}$ explored as the system cools, and predicts that the configurational entropy ${ }^{5,9,10}$ (a measure of the number of local potential energy minima sampled by the liquid) is related to the diffusion constant. Here we report a stringent test of the thermodynamic approach for liquid water (a convenient system to study because of an anomalous pressure dependence in the diffusion constant). We

$\dagger$ Present addresses: Polymers Division and Center for Theoretical and Computational Materials Science, National Institute of Standards and Technology, Gaithersburg, Maryland 20899, USA (F.W.S.); Dipartmento di Fisica e Istituto Nazionale per la Fisica della Materia, Università di Roma La Sapienza, P. le Aldo Moro 2, I-00185, Roma, Italy (A.S.) 